\title{
BCLA Pioneers Lecture - Evidence Basis for Patient Selection: How to Predict Contact Lens Success
}

\section{Prof James S Wolffsohn}

\author{
Aston University, Birmingham, UK
}

A prediction (Latin præ-, "before," and dicere, "to say") or forecast can be defined as "a statement about the way things will happen in the future, often but not always based on experience or knowledge. Although guaranteed information about the future is in many cases impossible, prediction is necessary to allow plans to be made about possible developments" (Wikipedia). Some predictions in the field of contact lenses have been very high profile and controversial, such as the predicted demise in rigid gas permeable contact lenses (RGPs) by Nathan Efron, ${ }^{1}$ whereas others, such as the presumed safety of multipurpose contact lens care solutions that have met specified regulatory criteria, have proved disastrously wrong (resulting in the Fusarium outbreak). ${ }^{2}$ However, as eye care practitioners, it is important that we use the evidence from studies to predict patient outcomes. This will reduce their risk of complications, aid in early diagnosis and allow patients to achieve the best possible refractive correction and management of their symptoms first time. Noninformed selection of visual correction such as a multifocal contact lens design, or treatment such as a tear supplement for dry eye, must lead to less than possible satisfaction in many patients. It is surmised that this leads to contact lens drop-out ${ }^{3}$ and patients living with suboptimal vision and comfort due to the perception that 'the' treatment doesn't work.

\section{Contact Lens Wear Drop-outs}

As with traditional lens materials, ${ }^{4}$ success with silicone hydrogel lens wear (deemed as continued wear after 6 months) in a recent study of 60 new lens wearers was predicted by non-invasive breakup time at fitting, and baseline symptoms also contributed to the sensitivity and specificity of detecting drop-out. ${ }^{5}$ Interesting in these lens wearing neophytes, even the high oxygen permeability of silicone hydrogel contact lenses were unable to maintain baseline levels of fluorescein tear breakup time, tear meniscus height, bulbar hyperaemia, lid-parallel conjunctival folds, corneal staining, conjunctival staining and lid wiper epitheliopathy. This study enhanced previous finding with first generation silicone hydrogels that dryness symptoms after 1 week of refitting from a traditional soft contact lens are associated with discontinuation of contact lens wear. ${ }^{6}$ The only other factor known to be associated with discontinuation from contact lenses are psychosocial factors. ${ }^{7}$ Hence assessment of dry eye in patients wanting to lens wear should be used as an indicator of when to strongly promote enhanced wetting lenses and to warn patient of potential issues, prompting a more frequent review schedule.

\section{Contact Lens Fit}

Graeme Young and his team at Visioncare Research in Farnham, UK has been the instrumental in determining our understanding of the lens fit. Examining retrospective data from multiple studies they identified that although subjectively rated clinical characteristics often conflicted in their indication whether a lens appeared loose or tight, push-up ease had the highest sensitivity for determining acceptable fit. Centration had poor sensitivity and horizontal lag was associated with a loose fit whereas vertical lag was associated with a tight fit. My colleagues and I objectively analysed all elements of soft contact lens fit demonstrating that the key independent determinants of lens 
movement were horizontal lag (nasal and temporal), movement on blink in up-gaze and push-up recovery speed. If the quantified values were modelled using these components, $91 \%$ of variance in lens movement could be accounted for with just these assessments and this only reduced to $82 \%$ if the components were graded on a simplified 3 point scale. ${ }^{8}$ Hence a schematic was developed to allow rapid and comprehensive recording of soft lens fit. Interestingly on average different lens base-curves had no effect on lens fit characteristics, although stiffer silicone hydrogel compared to traditional HEMA soft contact lenses generally had lower centration and a faster push-up speed of recovery. This finding is explained by peripheral corneal shape. Our studies have built on other, identifying that central corneal topography as measured by a keratometer has little relevance to soft contact lens fit, with this being partly explained by peripheral corneal shape and more by corneoscleral topography. ${ }^{9}$

For rigid gas permeable contact lenses (RGPs), eyelid position and tear meniscus height, as well as corneal topography influence lens $\mathrm{fit}^{10}$ and initial ocular discomfort predicts RGP drop-outs and adaptation time. ${ }^{11}$

\section{Contact Lens Induced Dry-Eye}

Individual clinical dry eye tests such as non-invasive tear break-up time, tear meniscus height, phenol red test, lid-parallel conjunctival folds (LiPCoF)and lid wiper epitheliopathy are moderately related to self-rated ocular surface symptoms (as evaluated by the Ocular Surface Disease Index), but the strongest predictor of contact lens induced dry eye was a combination of non-invasive break-up time and nasal LIPCOF. ${ }^{12}$ Contact Lens Dry Eye Questionnaire (CL-DEQ) scores have been shown in a large population study to predicted patients' wearing experience in the form of poorer comfort, shorter comfortable wearing time and increased use of dryness treatments. ${ }^{13}$

\section{Compliance and Contact Lens Complications}

Risk propensity, younger age and male gender all increase the likelihood of poor compliance, whereas years of lens wear and interestingly practitioners perception of compliance do not $!^{14}$ The incidence or corneal infiltrative events (CIE) with silicone hydrogels is linked to bacterial bio-burden and smoking, but not staining (although this was noted in approximately half of patients). For continuous wear, $\mathrm{CIE}$ were best predicted by previous corneal staining and limbal redness. ${ }^{15}$ In a hospital treated cohort, although the overall predictive value of risk factors for a given individual were low, the risk of a patient developing a CIE was associated with wearing modality/lens type (greatest risk for extended-wear hydrogel lenses), male gender, smoking, the absence of relevant ocular and general health problems, and the late winter months. ${ }^{16}$ Microbial keratitis risk factors consistently reported include extended wear, occasional overnight lens use, poor lens/case care and hand hygiene, smoking, younger age, males, socioeconomic class, self-reported poor general health, diabetes and thyroid disease, increased lens exposure in daily wear (number of days of wear per week worn), hypermetropia, obtaining lenses via the Internet or mail order and the early period of lens wear, but not lens material. ${ }^{17}$

\section{Presbyopic Contact Lenses}

When it comes to successful wear of presbyopic contact lenses, while many studies over the last decade have compared lenses mainly to monovision in crossover, one month adaptation studies, 
only one has tried to predict success. The author of this study concluded "These results suggest that making a prediction of 'success or not' based on consulting room acuity tests alone is probably unwise. ${ }^{18}$ More research is currently underway with modern presbyopic lens optics and more advanced clinical tests to see whether this is still the case.

Hence in conclusion, while there is still much research needed to clarify some of the key aspects of patient selection and preferences, eye care practitioners can use current evidence to refine their management of individual patients to enhance their clinical care and to promote long-term, safe contact lens wear.

\section{References}

1 Efron N. Obituary - rigid contact lenses. Contact Lens Ant Eye 2010;33:245-252.

2 Tu EY, Joslin CE. Recent outbreaks of atypical contact lens keratitis: what have we learnt. Am J Ophthalmol 2010;150:602-608.

3 Young G, Veys J, Pritchard N, Coleman S. A multi-centre study of lapsed contact lens wearers. Ophthalmic Physiol Opt 2002;22:516-527.

4 Glasson MJ, Stapleton F, Keay L, Sweeney D, Willcox DP. Differences in clinical parameters and tear film of tolerant and intolerant contact lens wearers. Invest Ophthalmol Vis Sci 2003;44:5116-5124.

5 Best N, Drury L, Wolffsohn JS. Predicting success with silicone-hydrogel contact lens in new lens wearers. Contact Lens Ant Eye 2013;36:232-237.

6 Schafer J, Mitchell GL, Chalmer RL, Long B, Dillehay S, Barr J, Bergenske P, Donshik P, Secor $\mathrm{G}$, Yoakum J. The stability of dryness symptoms with silicone hydrogel contact lenses. Eye Contact Lens 2007;33:247-252.

7 Jutai J, Day H, Woolrich W, Strong G. The predictability of retention and discontinuation of contact lenses. Optometry 2003;74:299-308.

8 Wolffsohn JS, Hunt OA, Basra AK. Simplified recording of soft contact lens fit. Contact Lens Ant Eye 2009;32:37-42.

9 Hall LA, Young G, Wolffsohn JS, Riley C. The influence of corneoscleral topography on soft contact lens fir. Invest Ophthalmol Vis Sci

10 Cardona G, Isern R. Topography-based RGP fitting in normal corneas: the relevance of eyelid and tear film attributes. Eye Contact Lens 2011;37:359-364.

11 Fujita H, Sano K, Sasaki S, Ohno-Matsui K, Tanaka T, Baba T, Mochizuki M. Ocular discomfort at the initial wearing of rigid gas permeable contact lenses. Jpn J Ophthalmol 2004;48: 376-379.

12 Pult $\mathrm{H}$, Purslow C, Murphy PJ. The relationship between clinical signs and dry eye symptoms. Eye 2011;25:502-510.

13 Young G, Chalmers RL, Napier L, Hunt C, Kern J. Characterizing contact lens-related dryness symptoms in a cross-section of UK soft lens wearers. Contact Lens Ant Eye 2011;34:64-70.

14 Carnt N, Keay L, Willcox M, Evans V, Stapleton F. Higher risk taking propensity of contact lens wearers is associated with less compliance. Contact Lens Ant Eye 2011;34:202-206. 
15 Szczotka-Flynn L, Lass JH, Sethi A, Debanne S, Benetz BA, Albright M, Gillespie B, Kuo J, Jacons MR, Rimm A. Risk factors for corneal infiltrative events during continuous wear of silicone hydrogel contact lenses. Invest Opthalmol Vis Sci 2010;51:5421-5430.

16 Morgan PB, Efron N, Brennan NA, Hill EA, Raynor MK, Tullo AB. Risk factors for the development of corneal infiltrative events associated with contact lens wear. Invest Ophthalmol Vis Sci 2005;46:3136-3143.

17 Stapleton F, Carnt N. Contact lens-related microbial keratitis: how have epidemiology and genetics helped us with pathogenesis and prophylaxis. Eye 2012;26:185-193.

18 Woods J, Woods CA, Fonn D. Early symptomatic presbyopes - what correction modality works best? Eye Contact Lens 2009;5:221-226. 